\title{
Real space delocalization, resonance, and aromaticity
}

\author{
Leonard Reuter \& Arne Lüchow*
}

When chemists want to explain a molecule's stability and reactivity, they often refer to the concepts of delocalization, resonance, and aromaticity. Resonance is commonly discussed within the electronic structure framework of valence bond theory as the stabilizing effect of mixing different Lewis structures. Yet, most computational chemists work with delocalized molecular orbitals, which are also usually employed to explain the concept of aromaticity, a special kind of ring delocalization that shows up in cyclic planar systems which abide certain number rules[1]. As an intuitive picture for aromaticity, an electronic ring current has been hypothesized[2, 3]. However, all three concepts lack a real space definition, that is not reliant on orbitals or specific wave function expansions. Here, we outline a redefinition from first principles: the concepts are of kinetic nature and related to saddle points of the all-electron probability density $|\Psi|^{2}$. Delocalization means that likely electron arrangements[4, 5] are connected via paths of high probability density in the many-electron real space. In this picture, resonance is the consideration of additional electron arrangements, which offer alternative paths of higher probability. Most notably, the concept of aromatic ring currents in absence of a magnetic field is rejected and the famous $4 n+2$ Hückel rule is derived from nothing but the antisymmetry of fermionic wave functions. The analysis developed in this work allows for a quantitative discussion of important chemical concepts that were previously only accessible qualitatively or restricted to specific electronic structure frameworks.

Why do neutral atoms bind? In the early twentieth century, the covalent bond formulated by Lewis proposed a conundrum, which even lead Lewis to believe, that "Coulomb's law ... must fail at small distances."[6] The first molecular application of Schrödingers wave equation[7] by Heitler and London[8] solved the puzzle mathematically, but lacked a physically understandable explanation. Hellmann[9] and later Ruedenberg[10] related the covalent bond to the-essentially kinetic'electron sharing', while others—starting with Slater[11]—saw a predominately electrostatic origin. 
While the kinetic picture prevailed for molecules with first-row atoms (e.g. $\mathrm{H}_{2}^{+}$and $\mathrm{H}_{2}$ ), it is disputed for larger systems[12, 13].

Pauling improved the Heitler-London picture of the chemical bond by giving even homoatomic bonds a certain ionic contribution, which gave rise to valence bond (VB) theory[14]. This mixing of ionic terms into the covalent wave function does not only lower the total energy-which is called resonance-, it also offers a coherent generalized picture of bonding, where covalent and ionic bonds are only the extreme forms of a continuously defined bond.

The stability of conjugated or aromatic systems is commonly attributed to the delocalization of $\pi$ electrons, which is closely related to Ruedenberg's 'electron sharing'. Hückel had an elegant orbital-based explanation for the at first sight bizarre difference between aromatic and antiaromatic systems, i.e. with $4 n+2$ and $4 n \pi$ electrons respectively[1]. This apparent simplicity-among other things - eventually lead to the rise of molecular orbital (MO) theory. The Hückel rules' seeming failure for some extended aromatic molecules like pyrene (Fig. 1)—which is not a failure of the Hückel method-is elegantly resolved with the concept of 'conjugated circuits' $[15,16,17]$ : the total aromaticity is ascribed to the existence of cyclic electronic subsystems, which abide the $4 n+2$ rule. Baird showed, that for triplet systems, the aforementioned Hückel rule is inverted, i.e. compounds with $4 n \pi$ electrons are aromatic[18].

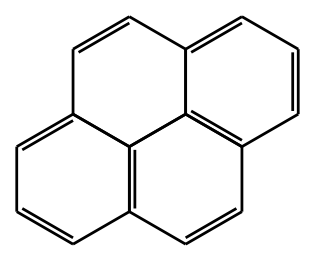

Figure 1: Pyrene, a planar aromatic system.

Already before Hückel's work, the diamagnetic properties of aromatic systems were attributed to electronic 'ring currents' $[2,19,20,3]$. Theoretical studies used this notion of ring currents to explain aromaticity also in absence of a magnetic field and discussed the role of the ionic structures[21, 22, 23, 24]. Notably, Shurki et al. stated that the "flow of the electrons around the $\mathrm{C}_{4} \mathrm{H}_{4}$ ring is, therefore, interrupted", which is close to the line of arguments we intend to develop in this article.

Bader and coworkers developed the 'delocalization index' (DI) as a real space measure of delocalization $[25,26]$ in the framework of the quantum theory of atoms in molecules (QTAIM).[27, 28] It is based on the one- and two-electron densities $\rho(\mathbf{r})$ and $\rho_{2}\left(\mathbf{r}, \mathbf{r}^{\prime}\right)$. Poater et al. subsequently 
introduced the "mean of all DIs of para-related carbon atoms in a given six-membered ring (PDI)" as a measure of aromaticity.[29] However, as Martín Pendás and Francisco and the authors of this work showed recently, the delocalization index is better understood as a measure of ionicity.[30, 5]

Probability density analysis (PDA) $[4,5]$ was introduced as the all-electron equivalent of QTAIM. PDA recovers Lewis structures by analyzing local maxima of $|\Psi|^{2}$, which we label structure critical points (SCPs). Here, we add the $3 N$ dimensional saddle points, labeled delocalization critical points (DCPs), to the analysis. A DCP is the lowest point on the maximum probability path (MPP) between two adjacent SCPs, much like the lowest point on a mountain ridge.

This mathematical framework allows for the probabilistic quantification of Ruedenberg's 'electron sharing', which is an increased freedom of movement (i.e. delocalization) lowering the kinetic energy_cf. increasing the box length for a particle in a box.

For $\mathrm{H}_{2}^{+}$, the two SCPs are simply the two proton positions (PDA and QTAIM are identical for one-electron systems). For infinitely separated protons, $|\Psi|^{2}$ at the DCP approaches zero, while it is non-zero for the bound state ${ }^{2} \Sigma_{g}^{+}$at equilibrium distance (Fig. 2). For the first exited state ${ }^{2} \Sigma_{u}^{+},|\Psi|^{2}$ at
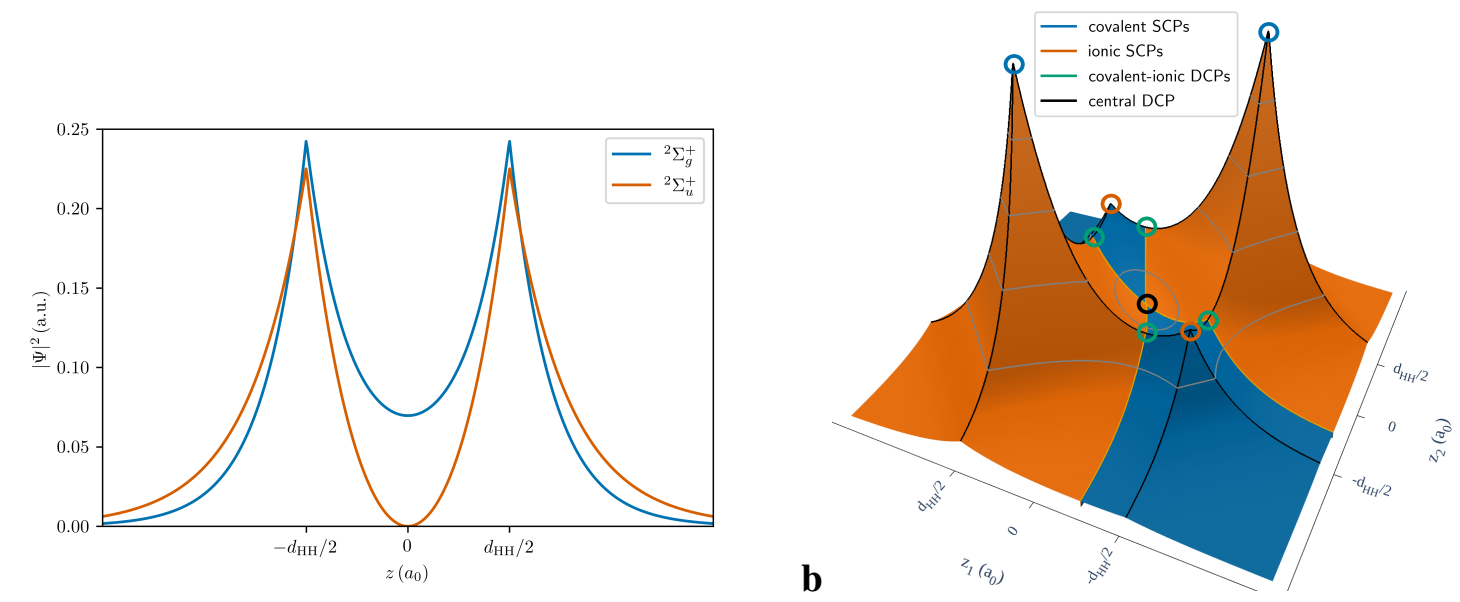

Figure 2: $|\Psi|^{2}$ with the electrons on the bond axis $z \cdot \mathbf{a ~}_{2}^{+}$at the equilibrium distance $\left(d_{\mathrm{HH}}=\right.$ $\left.2.00 a_{0}[31]\right) . \mathbf{b ~ H} \mathrm{H}_{2}$ at the equilibrium distance $\left(d_{\mathrm{HH}}=1.40 a_{0}\right)$.

the DCP equals zero (node) independent of the proton-proton distance. For the infinitely dissociated system as well as for the ${ }^{2} \Sigma_{u}^{+}$state, the electron is thus not shared, since-while mathematically being at both protons- the electron could never move from one nucleus to the other. This impossibility of crossing a node of $|\Psi|^{2}$ is well established in the context of potential barriers, where an infinitely large barrier prevents particles from tunneling. In order to discuss barriers instead of low probability density, we define a probabilistic potential $\Phi$, which is monotonically decreasing with $|\Psi|^{2}$. It is the scalar potential of the drift velocity $\mathbf{u}$, which appears in the diffusion Monte Carlo method[32] as well 
as in stochastic quantum mechanics[33].

$$
\Phi=-\frac{\hbar}{2 m_{e}} \ln |\Psi|^{2}, \quad \mathbf{u}=-\nabla \Phi=\frac{\hbar}{m_{e}} \frac{\nabla \Psi}{\Psi}
$$

A probabilistic barrier between two SCPs can thus be defined as the value of $\Phi$ at the highest DCP on the connecting path (this barrier approaches infinity, if $|\Psi|^{2}$ at the DCP vanishes).

A VB wave function in the minimal basis of orbitals $\varphi_{\mathrm{K}}$ is chosen to investigate the two-electron bond in $\mathrm{H}_{2}$, (Eq. 2).

$$
\Psi=N\left[(1-\eta) \psi_{\text {cov. }}+\eta \psi_{\text {ion. }}\right], \quad \varphi_{\mathrm{K}}(\mathbf{r})=\sqrt{\frac{\zeta^{3}}{\pi}} e^{-\zeta\left|\mathbf{r}-\mathbf{r}_{\mathrm{K}}\right|}
$$

The coefficient $\eta$ controls the ionic contribution and the exponent $\zeta$ the contraction of the wave function at the protons. Note, that this ansatz is equivalent to $\operatorname{CASSCF}(2,2)$. Four structure critical points and five delocalization critical points are identified with PDA. For all of these points, both electrons are positioned on the bond axis, (Fig. 2b). The four SCPs are the covalent and ionic arrangements of the electrons. The central DCP is a second-order saddle point connecting the two covalent SCPs as well as the two ionic SCPs. It describes a concerted two-electron exchange. With a correlated wave function, the electrons would avoid each other, moving the central DCP away from the depicted plane. The four covalent-ionic DCPs describe the one-electron move from a covalent to an ionic arrangement or vice-versa. There are apparently two paths from one covalent SCP to the other: the concerted two-electron exchange via the central DCP and the step-wise electron exchange via two covalent-ionic DCPs and an ionic SCP. Since $|\Psi|^{2}$ is lower at the central DCP compared to the ionic-covalent DCP, the probabilistic barrier (i.e. the largest probabilistic potential $\Phi$ ) is larger for the concerted two-electron exchange, (Fig. 3a). The ionic arrangements thus serve a purpose which is analogous to a reaction intermediate. The probabilistic barriers is minimal for $\eta=0.42$, which is close to the Hartree-Fock wave function $(\eta=1 / 2)$. This is consistent with the established view, that “... the electrons in the HF-MO description of $\mathrm{H}_{2}$ are completely delocalized."[24]

In order to validate the proposed connection between resonance and the probabilistic barrier, the kinetic $(T)$ and potential $(V)$ contributions to the total stabilization have to be investigated. For $\mathrm{H}_{2}$, the resonance energy is defined as the energetic difference between the purely covalent $(\eta=0)$ Heitler-London wave function and the optimized $(\eta=0.21)$ VB wave function. Since the orbital 

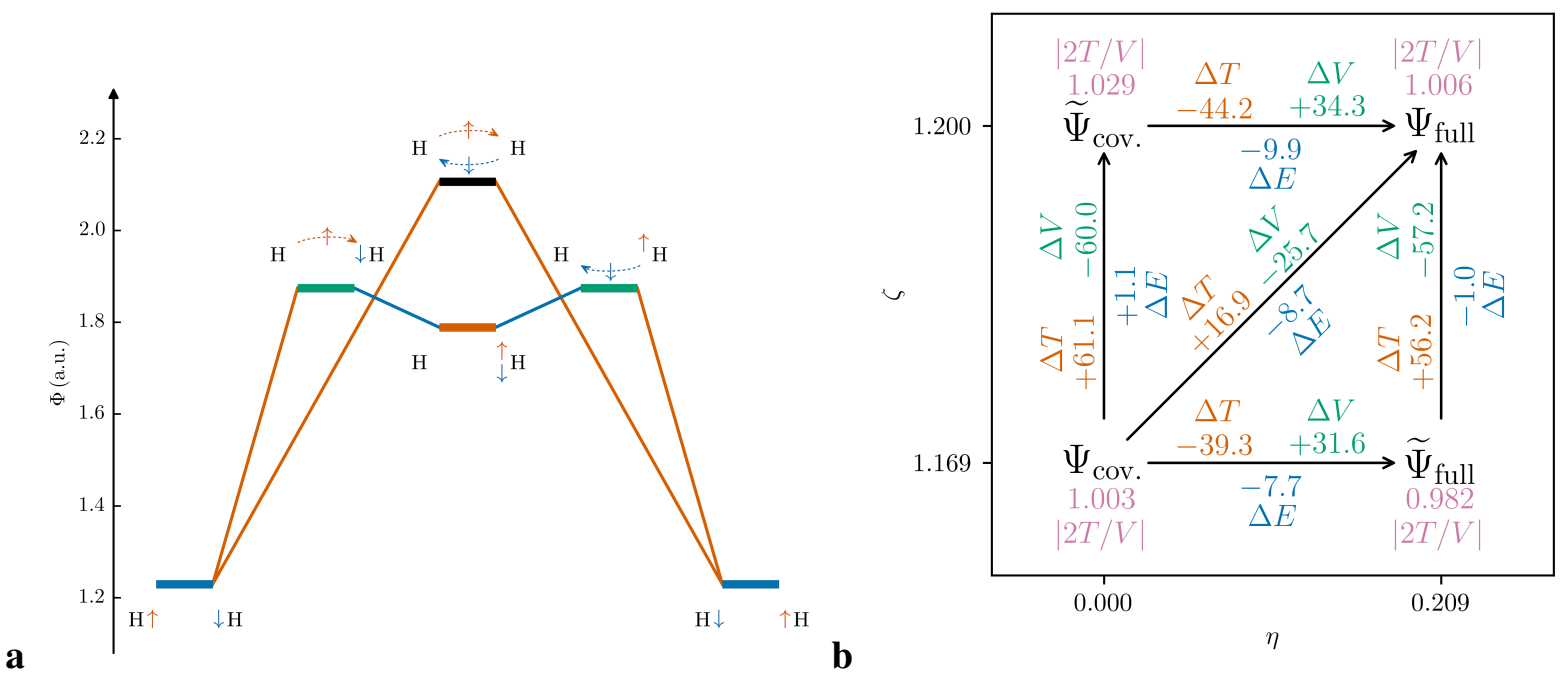

Figure 3: $\mathrm{H}_{2}$ at the equilibrium distance $\left(1.4 a_{0}\right)$. a Probabilistic barriers for the concerted twoelectron exchange and for the step-wise exchange with an ionic intermediate SCP. b Step-wise morphing of the covalent Heitler-London wave function $\Psi(\eta=0, \zeta=1.169)$ into the full VB wave function $\Psi(\eta=0.209, \zeta=1.200)$. All energies in $\mathrm{m} E_{h}$.

coefficients $\zeta$ have been optimized, the virial ratio is close to one $(|2 T / V|<1.007)$ for both functions. It is not exactly equal to one, since the proton-proton distance has not been relaxed. Due to this nonrelaxation, the virial ratio of the resonance energy is quite large: $|2 \Delta T / \Delta V|=1.320$. However, the energy difference is obviously still dominated by a decreasing potential and an increasing kinetic energy, qualitatively following the virial theorem. Yet, it can be shown-analogously to the 'stepwise morphing' by Ruedenberg et al.[10, 31] — that resonance in $\mathrm{H}_{2}$ is a kinetic stabilization.

Resonance can be divided into two 'physical' steps: contraction of the wave function at the nuclei and the actual resonance (i.e. mixing in of the ionic contribution), (Fig. 3b). There are two ways of describing the step-wise morphing: mixing followed by contraction or contraction followed by mixing. We will proceed with the former, since the contraction is destabilizing for the latter. The total energy difference of $-8.7 \mathrm{~m} E_{h}$ can be attributed to $89 \%$ to the mixing, which, for its part, can be attributed to a kinetic stabilization $\left(-39.3 \mathrm{~m} E_{h}\right)$ outweighing an increase of the potential energy $\left(+31.6 \mathrm{~m} E_{h}\right)$. The remaining $11 \%$ resulting from orbital contraction lead-of course-to an overall increase of the kinetic energy. Yet, the nature of resonance remains essentially kinetic, justifying the discussion of the probabilistic barrier as the fundamental principle of resonance.

Going beyond singlet two-electron systems, the antisymmetry of fermionic wave functions has to be considered: the exchange of two same-spin electrons changes the sign of the wave function $\Psi$, while the probability density $|\Psi|^{2}$ remains the same, preserving the elecrons' indistinguishability. 
For an hypothetical triplet two-electron bond, having an antisymmetric spatial function, the picture thus changes drastically. There are only two SCPs (the covalent ones) and upon exchange of the two electrons, the sign of $\Psi$ changes. Therefore, according to the intermediate value theorem, it is necessarily zero somewhere on any path connecting the two covalent SCPs, yielding an infinitely high probabilistic barrier. This is an easy explanation for why triplet $\mathrm{H}_{2}$ is not binding: the electrons are not shared.

Going beyond two electrons, the same arguments can still be used to predict and explain delocalization. For $n$ same-spin electrons equally spaced on a ring, the rotation of the ring by $2 \pi / n$ is described by the cyclic permutation $\sigma_{n}=\left(\begin{array}{lll}2 & 3 \ldots n & \ldots\end{array}\right) \in S_{n}$. This permutation is positive for odd $n$ and negative for even $n$. If the sign of a permutation is negative, the sign of $\Psi$ changes upon its application. If it is positive, $\Psi$ is unchanged. Thus, again arguing with the intermediate value theorem, the rotation of an even-numbered ring of same-spin electrons is impossible, since it has to pass $|\Psi|^{2}=0$ (i.e. an infinitely large probabilistic barrier). This discussion of parity is related to the 'electron-switch symmetry index' by Shurki et al.[24]

If two rings (spin-up and spin-down) are combined (cf. the alternating spin structure of benzene), the intermediate value theorem apparently does not help anymore: rotating two rings of the same size at once always preserves the sign of $\Psi$ independent of the parity. However, for a Hartree-Fock (HF) wave function there would still be a node crossing: the HF wave function can be written as a product of a spin-up and a spin-down determinant[32]. For both determinants individually, the intermediate value theorem can still be applied, making the rotation of two rings possible only if both have an odd number of electrons. While HF wave functions do not solve the Schrödinger equation, they are often (in some respect) qualitatively correct-especially for aromatic systems. The exact wave function can be expressed as a sum of determinants, each of which is an eigenfunction of $\hat{S}_{z}$ and can also be written as a product of two determinants. While the intermediate value argument holds for each of the determinants, they have different nodes and are not all 0 at the same all-electron position. However, paths, that are forbidden at Hartree-Fock level are are still presumably unlikely at a correlated level, since the Hartree-Fock determinant often dominates the exact wave function expansion.

The numbers of both, spin-up and spin-down electrons thus have to be odd in order to have circular delocalization: $N_{\uparrow}, N_{\downarrow} \in\{2 n+1: n \in \mathbb{N}\}$. This can be called the 'two times $2 n+1$ ' rule. If the model of electrons on a ring is applied to the $\pi$ system of singlet planar ring molecules (i.e. $N_{\uparrow}=N_{\downarrow}$ ), 
the Hückel rule follows directly as $2(2 n+1)=4 n+2$. For $M_{S}= \pm 1$, which determine the symmetry of the triplet state, the Baird rule is retrieved: $N_{\uparrow}=N_{\downarrow} \pm 2$ leads to the two equivalent rules $4 n+4$ and $4 n$.

This real space view of delocalization can also describe the aromaticity of polycyclic aromatic compounds like pyrene (Fig. 1), for which the Hückel rule is not valid. While the total number of $\pi$ electrons is 16 , there is still a plethora of possible rotations: e.g. on each six-membered ring (6 electrons), along two six-membered rings (10 electrons), or including all electrons on the edges of the system (14 electrons). All these possible rotations lead to a stabilizing delocalization of the $\pi$ electrons. This picture of polycyclic aromatic compounds is the real space analogue of "conjugated circuits'.[15, 16, 17]

The two times $2 n+1$ rule is not restricted to circular planar systems as the permutations' signs are independent of the spatial arrangement. As has been shown, it also applies to $\mathrm{H}_{2}$. Furthermore, the concept of 'paired electrons' is to some degree justified by and connected to the real space picture presented in this work: the electron pair is the smallest multi-electron system abiding the 'two times $2 n+1$ ' rule.

While singlet cyclobutadiene (CBD) is considered to be antiaromatic, the $D_{6 h}$ symmetric benzene is aromatic according to the Hückel rule. With $\pi$-only wave functions, the two alternating covalent spin structures are the most important (by PDA weight[5]) for both systems in planar geometry. In order to explore delocalization, the maximum probability paths connecting these structures thus have to be investigated. This is somewhat analogous to work by Shurki et al.[24] and seemingly related to the concept of diamagnetic ring currents[20, 3]. However, like Maynau and Malrieu[23] we do not restrict our search to paths, where all electrons move in the same circular direction (Fig. 4a). Instead, the spin-up and spin-down electrons can also rotate in opposite directions (Fig. 4b), which will eventually turn out to be more likely. Staying with the picture of a ring current, the electrons can move between the covalent spin structures $\mathbf{1}$ and 7, passing step-wise through the ionic SCPs 2-6a without any concerted movement. A restriction of this same-direction path to covalent SCPs enforces a concerted movement of six electrons. Assuming, that the spin-up and spin-down electrons rotate in opposite directions, the step-wise movement passes the two non-alternating covalent SCPs $\mathbf{3 b}$ and $\mathbf{5 b}$ in addition to the ionic SCPs $\mathbf{2}, \mathbf{4 b}$, and $\mathbf{6 b}$. Here, a restriction to covalent spin structures only leads to concerted two-electron movements. All four investigated paths (same/opposite direction, restricted 


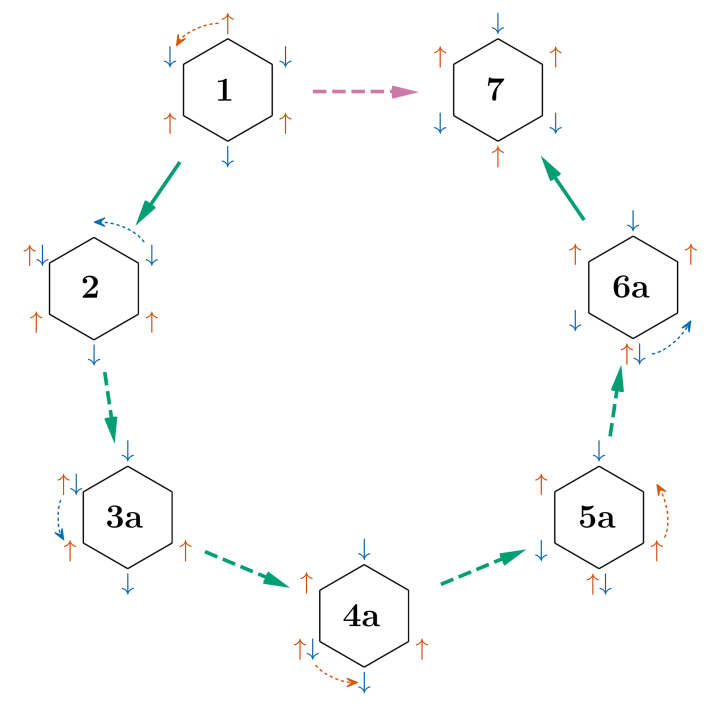

$\mathbf{a}$

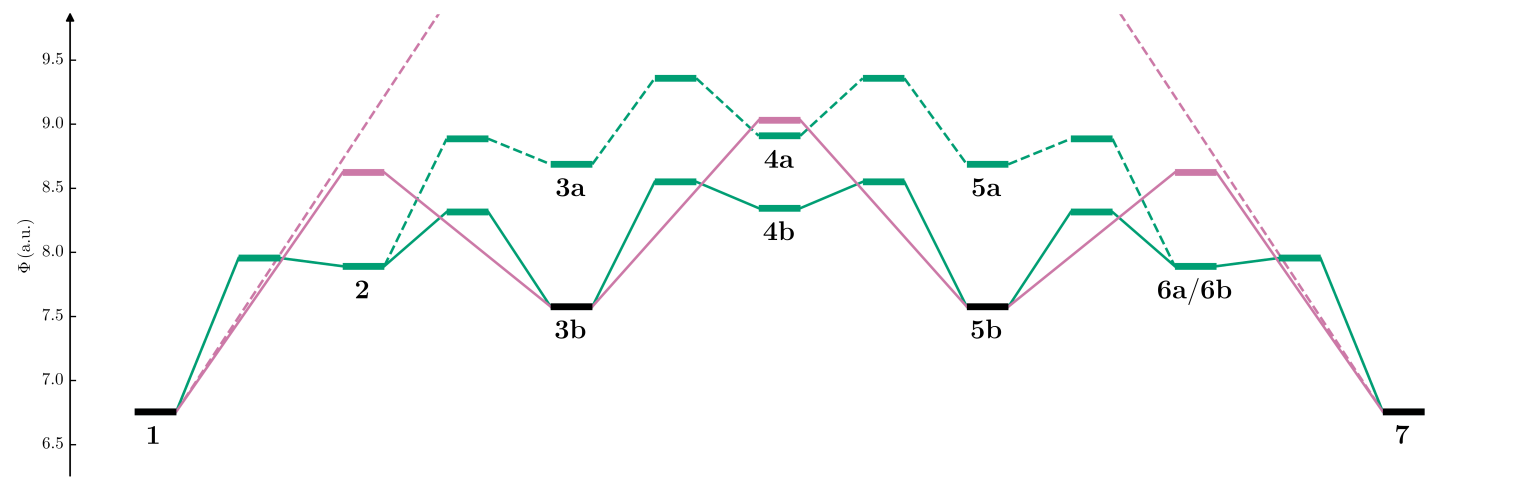

b

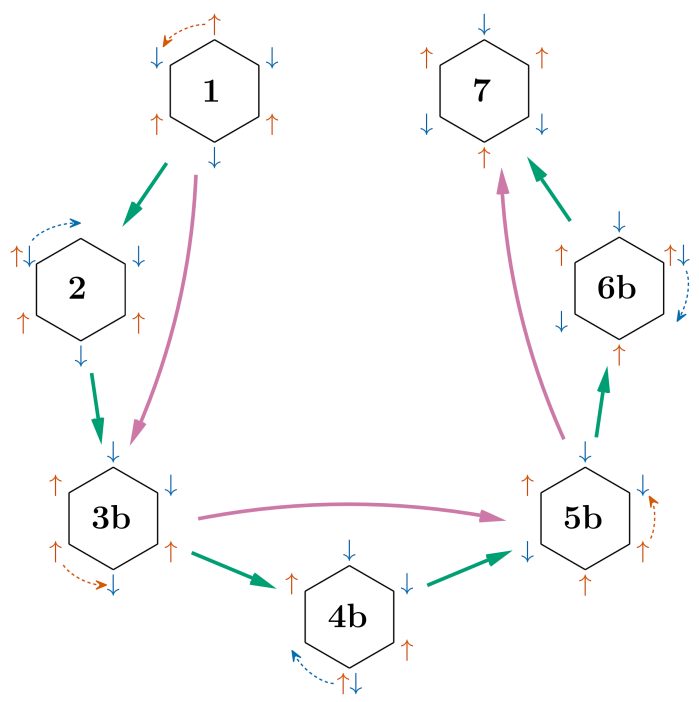

c

Figure 4: Maximum probability paths for the $\pi$ system of benzene: without restrictions (green) and restricted to covalent DCPs (purple). Spin-up and spin-down electrons rotate in a the same direction, b opposite directions. c Probabilistic barriers.

to covalent SCPs/no restriction) are compared regarding the probabilistic barrier (Fig. 4c).

In agreement with the previously discussed qualitative picture (two-times $2 n+1$ rule), none of these paths crosses a node, i.e. there is no infinitely large barrier. In contrast to the prevalent picture of diamagnetic ring currents, the opposite direction paths are more likely, having a lower probabilistic barrier. Yet, it is easily imaginable, that an external magnetic field inverts this relation. These results contradict an earlier comparison[23], which however only took concerted six-electron movements in account. The importance of ionic structures is in good agreement with the results by Shurki et al.[24] for the same direction movement. In general, the role of the ionic spin structures is, however, not qualitatively different from the role of the ionic structure in $\mathrm{H}_{2}$, since the dominant opposite direction movement is much less affected by the restriction to covalent structures.

For singlet $\mathrm{CBD}$ - again in agreement with the qualitative picture-the movement between the two alternating spin structures is not possible: there is a node crossing independent of any ionic 
contribution. As Shurki et al. already stressed, the ionic structures thus have a low weight and their mixing is only slightly stabilizing, since they do not enhance delocalization between covalent structures.

For the $D_{4 h}$ symmetric triplet CBD (which is aromatic according to the Baird rule), MPPs can be calculated like for benzene (Fig. 5). As for benzene, the opposite direction movement is preferred over the same direction movement.

a

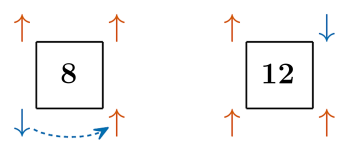

Figure 5: Maximum probability paths analysis for the $\pi$ system of triplet CBD with $M_{S}=+1 \hbar$ : without restrictions (green) and restricted to covalent DCPs (purple). a Spin-up and spin-down electrons rotate in opposite directions. b Probabilistic barriers.

The freedom of movement for electrons is restricted by borders of low or even zero all-electron probability density $|\Psi|^{2}$.

Due to antisymmetry, in the HF wave function, delocalization is only possible for an odd number of same-spin electrons. For correlated wave functions, impossibility of delocalization becomes low possibility, but odd numbers of same-spin electrons are still favored. This leads directly to the Hückel and Baird rules of aromaticity ( $4 n+2$ and $4 n$ respectively) as well as to the electron pair.

In $\mathrm{H}_{2}$, the kinetic nature of resonance has been demonstrated. For $\mathrm{H}_{2}$ and the $\pi$ systems in benzene and triplet cyclobutadiene, the ionic structures act as an intermediate analogue and play an important role in the broad phenomenon of delocalization.

A Ruedenberg analysis (i.e. step-wise morphing) has shown, that this connection between the saddle points of $|\Psi|^{2}$ and bonding can explain the nature of resonance: the mixing of ionic contributions is essentially a kinetic stabilization.

The in-depth analysis of aromaticity in benzene and triplet cyclobutadiene revealed that the opposite direction movement of spin-up and spin-down electrons is more important than the same direction movement. This leads us to reject the notion of diamagnetic current in absence of a magnetic field. 


\section{References}

[1] Hückel, E. Quantentheoretische Beiträge zum Benzolproblem. I. Die Elektronenkonfiguration des Benzols und verwandter Verbindungen. [Quantum theoretical contributions to the benzene problem. I. The electron configuration of benzene and related compounds]. Z. Phys. 70, 204-286 (1931).

[2] Raman, C. V. \& Krishnan, K. S. Magnetic double-refraction in liquids. Part I.-Benzene and its derivatives. Proc. R. Soc. Lond. A 113, 511-519 (1927).

[3] Gomes, J. A. N. F. \& Mallion, R. B. Aromaticity and ring currents. Chem. Rev. 101, 1349-1384 (2001).

[4] Lüchow, A. Maxima of $|\Psi|^{2}$ : a connection between quantum mechanics and Lewis structures. J. Comput. Chem. 35, 854-864 (2014).

[5] Reuter, L. \& Lüchow, A. On the connection between probability density analysis, QTAIM, and VB theory. Phys. Chem. Chem. Phys. 22, 25892-25903 (2020).

[6] Lewis, G. N. The atom and the molecule. J. Am. Chem. Soc. 38, 762-785 (1916).

[7] Schrödinger, E. Quantisierung als Eigenwertproblem. [Quantization as an eigenvalue problem]. Ann. Phys. 384, 361-376 (1926).

[8] Heitler, W. \& London, F. Wechselwirkung neutraler Atome und homöopolare Bindung nach der Quantenmechanik. [Interaction of neutral atoms and homopolar bond according to quantum mechanics]. Z. Phys. 44, 455-472 (1927).

[9] Hellmann, H. Zur Rolle der kinetischen Elektronenenergie für die zwischenatomaren Kräfte. [On the role of the kinetic electron energy for the interatomic forces]. Z. Phys. 85, 180-190 (1933).

[10] Ruedenberg, K. The physical nature of the chemical bond. Rev. Mod. Phys. 34, 326-376 (1962).

[11] Slater, J. C. The virial and molecular structure. J. Chem. Phys. 1, 687-691 (1933).

[12] Schmidt, M. W., Ivanic, J. \& Ruedenberg, K. Covalent bonds are created by the drive of electron waves to lower their kinetic energy through expansion. J. Chem. Phys. 140, 204104 (2014). 
[13] Levine, D. S. \& Head-Gordon, M. Clarifying the quantum mechanical origin of the covalent chemical bond. Nat. Commun. 11, 4893 (2020).

[14] Pauling, L. The nature of the chemical bond. Applications of results obtained from the quantum mechanics and from a theory of paramagnetic susceptibility to the structure of molecules. J. Am. Chem. Soc. 53, 1367-1400 (1931).

[15] Herndon, W. C. Resonance energies of aromatic hydrocarbons. A quantitative test of resonance theory. J. Am. Chem. Soc. 95, 2404-2406 (1973).

[16] Swinborne-Sheldrake, R., Herndon, W. C. \& Gutman, I. Kekulé strutures and resonance energies of benzenoid hydrocarbons. Tetrahedron Lett. 16, 755-758 (1975).

[17] Randić, M. Conjugated curcuits and resonance energies of benzoid hydrocarbons. Chem. Phys. Lett. 38, 68-70 (1976).

[18] Baird, N. C. Quantum organic photochemistry. II. Resonance and aromaticity in the lowest ${ }^{8} \pi \pi^{*}$ state of cyclic hydrocarbons. J. Am. Chem. Soc. 94, 4941-4948 (1972).

[19] Lonsdale, K. Diamagnetic and paramagnetic anisotropy of crystals. Rep. Prog. Phys. 4, 368-389 (1937).

[20] Elvidge, J. A. \& Jackman, L. M. Studies of aromaticity by nuclear magnetic resonance spectroscopy. Part I. 2-Pyridones and related systems. J. Chem. Soc. 859 (1961).

[21] Brooks, H. Diamagnetic anisotropy and electronic structure of aromatic molecules. J. Chem. Phys. 8, 939-949 (1940).

[22] McWeeny, R. The diamagnetic anisotropy of large aromatic systems V: interpretation of the results. Proc. Phys. Soc. A 66, 714-720 (1953).

[23] Maynau, D. \& Malrieu, J. P. A valence bond effective Hamiltonian for the neutral states of $\pi$ systems. 2. Results. J. Am. Chem. Soc. 104, 3029-3034 (1982).

[24] Shurki, A., Hiberty, P. C., Dijkstra, F. \& Shaik, S. Aromaticity and antiaromaticity: what role do ionic configurations play in delocalization and induction of magnetic properties? J. Phys. Org. Chem. 16, 731-745 (2003). 
[25] Bader, R. F. W., Streitwieser, A., Neuhaus, A., Laidig, K. E. \& Speers, P. Electron delocalization and the Fermi hole. J. Am. Chem. Soc. 118, 4959-4965 (1996).

[26] Fradera, X., Austen, M. A. \& Bader, R. F. W. The Lewis model and beyond. J. Phys. Chem. A 103, 304-314 (1999).

[27] Bader, R. F. W. \& Beddall, P. M. Virial field relationship for molecular charge distributions and the spatial partitioning of molecular properties. J. Chem. Phys. 56, 3320-3329 (1972).

[28] Bader, R. F. W. \& Nguyen-Dang, T. Quantum theory of atoms in molecules-Dalton revisited. In Advances in Quantum Chemistry, 63-124 (Academic Press, Inc., 1981), 14 edn.

[29] Poater, J., Fradera, X., Duran, M. \& Solà, M. The delocalization index as an electronic aromaticity criterion: application to a series of planar polycyclic aromatic hydrocarbons. Chem. Eur. J. 9, 400-406 (2003).

[30] Martín Pendás, A. \& Francisco, E. Decoding real space bonding descriptors in valence bond language. Phys. Chem. Chem. Phys. 20, 12368-12372 (2018).

[31] Ruedenberg, K. \& Schmidt, M. W. Why does electron sharing lead to covalent bonding? A variational analysis. J. Comput. Chem. 28, 391-410 (2007).

[32] Reynolds, P. J., Ceperley, D. M., Alder, B. J. \& Lester, W. A. Fixed-node quantum Monte Carlo for molecules. J. Chem. Phys. 77, 5593-5603 (1982).

[33] Nelson, E. Derivation of the Schrödinger equation from Newtonian mechanics. Phys. Rev. 150, 1079-1085 (1966). 


\section{Methods}

\section{Hydrogen molecules}

The equilibrium distances for $\mathrm{H}_{2}^{+}$and $\mathrm{H}_{2}$ are taken from Ruedenberg and Schmidt[37] and Huber and Herzberg[38], respectively. The wave functions for the two states $\left({ }^{2} \Sigma_{g}^{+}\right.$and $\left.{ }^{2} \Sigma_{g}^{-}\right)$of $\mathrm{H}_{2}^{+}$is built from the 1s orbitals of Eq. 2:

$$
\Psi_{2 \Sigma_{g}^{ \pm}}(\mathbf{r})=\frac{1}{\sqrt{2 \pm 2 S}}\left[\varphi_{\mathrm{A}}(\mathbf{r}) \pm \varphi_{\mathrm{B}}(\mathbf{r})\right], \quad S=\left\langle\varphi_{\mathrm{A}} \mid \varphi_{\mathrm{B}}\right\rangle
$$

The covalent and ionic VB structures of $\mathrm{H}_{2}$ are defined as

$$
\begin{aligned}
& \psi_{\text {cov. }}\left(\mathbf{r}_{1}, \mathbf{r}_{2}\right)=\frac{1}{\sqrt{2+2 S^{2}}}\left[\varphi_{\mathrm{A}}\left(\mathbf{r}_{1}\right) \varphi_{\mathrm{B}}\left(\mathbf{r}_{2}\right)+\varphi_{\mathrm{B}}\left(\mathbf{r}_{1}\right) \varphi_{\mathrm{A}}\left(\mathbf{r}_{2}\right)\right] \\
& \psi_{\text {ion. }}\left(\mathbf{r}_{1}, \mathbf{r}_{2}\right)=\frac{1}{\sqrt{2+2 S^{2}}}\left[\varphi_{\mathrm{A}}\left(\mathbf{r}_{1}\right) \varphi_{\mathrm{A}}\left(\mathbf{r}_{2}\right)+\varphi_{\mathrm{B}}\left(\mathbf{r}_{1}\right) \varphi_{\mathrm{B}}\left(\mathbf{r}_{2}\right)\right]
\end{aligned}
$$

The VB calculations were performed with XMVB[39, 40], all integrals were computed with Gaussian16[41].

\section{Benzene and cyclobutadiene}

Complete active space self-consistent field (CASSCF) geometry optimizations with an active space of all $\pi$ orbitals have been performed with Molpro[42] for benzene and triplet CBD. Each function of the Slater-type TZPae[43] basis has been expanded into 14 primitive Gaussian-type functions[44, 45]. The investigated multi-reference, $\pi$-only wave functions have been obtained from the CASSCF expansion: the determinants are reduced to the $\pi$ orbitals, while spin coupling and configuration interaction coefficients are left untouched. This way, the $\pi$ system is optimized in full presence of the $\sigma$ and core electrons, which is more accurate than any effective $\pi$ Hamiltonian. Note, that there is no need to calculate energies from these $\pi$-only wave functions while performing PDA.

\section{Probability density analysis}

The probability density analysis was performed with the quantum Monte Carlo code Amolqc. The saddle points of $|\Psi|^{2}$ (DCPs) were identified by applying the Newton method to $\Phi$. The maxima of $|\Psi|^{2}$ (SCPs) were identified with the steepest descent and L-BFGS[46] methods. 


\section{Data availability}

All relevant data are available in the supporting information file.

\section{Code availability}

The algorithms used for the findings are described in the methods section. The code Amolqc as well as any program used for evaluating data, starting other programs or making figures are available upon reasonable request.

\section{References}

[37] Ruedenberg, K. \& Schmidt, M. W. Why does electron sharing lead to covalent bonding? A variational analysis. J. Comput. Chem. 28, 391-410 (2007).

[38] Huber, K. P. \& Herzberg, G. Molecular spectra and molecular structure (Springer US, Boston, MA, 1979).

[39] Song, L., Mo, Y., Zhang, Q. \& Wu, W. XMVB: a program for ab initio nonorthogonal valence bond computations. J. Comput. Chem. 26, 514-521 (2005).

[40] Chen, Z. et al. XMVB 2.0: a new version of Xiamen valence bond program. Int. J. Quantum Chem. 115, 731-737 (2015).

[41] Frisch, M. J. et al. Gaussian 16 Revision C.01 (2016).

[42] Werner, H.-J. et al. MOLPRO, version 2019.2, a package of ab initio programs (2019).

[43] van Lenthe, E. \& Baerends, E. J. Optimized Slater-type basis sets for the elements 1-118. J. Comput. Chem. 24, 1142-1156 (2003).

[44] O-ohata, K., Taketa, H. \& Huzinaga, S. Gaussian expansions of atomic orbitals. J. Phys. Soc. Jpn. 21, 2306-2313 (1966).

[45] Petersson, G. A., Zhong, S., Montgomery, J. A. \& Frisch, M. J. On the optimization of Gaussian basis sets. J. Chem. Phys. 118, 1101-1109 (2003). 
[46] Nocedal, J. Updating quasi-Newton matrices with limited storage. Math. Comput. 35, 773-773 (1980).

Author contributions A.L. and L.R. conceived the project. A.L. is the main contributor of the QMC program Amolqc. L.R. added the necessary new code to Amolqc, performed all calculations, and prepared the figures and the manuscript with input from A.L.

Competing interests The authors declare no competing interests.

Correspondence and requests for materials should be addressed to A.L. 